\title{
Klebsiella liver abscess with multiple septic emboli
}

\author{
Jameela Al Salman, Rawan Al Agha \\ Internal Medicine Department, Salmaniya Medical Complex, Manama, Bahrain \\ Correspondence: Jameela Al Salman. Address: Internal Medicine Department, Salmaniya Medical complex, P.O.Box12, \\ Manama, Bahrain. Email: jalsalman@hotmail.com \\ Received: March 29, 2015 \\ DOI : $10.5430 /$ crim.v2n3p8 \\ Accepted: May 4, $2015 \quad$ Online Published: May 11, 2015
URL: http://dx.doi.org/10.5430/crim.v2n3p8
}

\section{Abstract}

A 29-years-old Bahraini presented to accident and emergency department with history of shortness of breath, cough and fever of 10 days duration. He was admitted for further evaluation. As an in-patient the condition worsened in respect to vision, headache, fever and respiration. A full computed tomography was done and showed multiple lung nodules and a liver abscess which was drained and cultured Klesiella pneumoniae. He was started on multiple antibiotics to treat the liver abscess but unfortunately the symptoms were worsening due to infective emboli to multiple body organs including the eyes and brain. The patient did not complete the treatment and preferred to travel overseas for second opinion. This case report is considered the first case report of Klebsiella liver abscess in the region of the Arabian Gulf and that is why it is worth reporting to grasp the attention to it as further cases may emerge.

\section{Keywords}

Abscess, Klebsiella, Antibiotics, Embolism, Liver

\section{Introduction}

Klebsiella pneumoniae is a gram negative, non-motile, facilitative anaerobic rod bacterium. It is found as a normal inhabitant in the skin, intestine and mouth but it can cause destructive changes to the lung tissue if aspirated. The most common condition caused by Klebsiella pneumoniae is pneumonia and more typically bronchopneumonia and bronchitis. The mortality with this infection can reach up to $50 \%$ despite treatment and up to $100 \%$ with bacteremia ${ }^{[1]}$. However, it can cause a range of diseases including urinary tract infection, soft tissue infection, diarrhea and meningitis.

Klebsiella mostly seen in the immunocompromised people or those with impaired respiratory defense including diabetes, chronic obstructive pulmonary disease and malignancy. It usually affects elderly and middle aged adults ${ }^{[2]}$.

More recently a new form is emerging which is Klebsiella pneumoniae liver abscess (KPLA). It is reported with increasing frequency in the Far East countries in the last three decades, especially in Taiwan and Korea but gradually emerging as global problem. Diabetes is known to predispose patients to this infection and its complications including: meningitis, bacteremia, endophthalmitis and necrotizing fascititis. In addition, serotypes of K1 and K2 further increase the virulence of the organism by impairing the phagocytosis process ${ }^{[3]}$. The organism tends to respond to prolonged course of antibiotics particularly Cephalosporin along with drainage of the abscess. 
A case of KPLA was diagnosed recently in a patient in the Kingdom of Bahrain. It is considered the first reported case in the Arabian Gulf region. This case is reported in the aim of familiarizing the physicians with the presentation of this uncommon case, its complications and management.

\section{Case presentation}

Mr. A. M. is a 29-year-old Bahraini male not known case of any medical illness presented to the accident and emergency department at Salmaniya Medical Complex-SMC (Manama/ Bahrain) on 17th of January 2015 with history of shortness of breath, cough, fever and right eye redness of ten days duration.

The patient was in Milan/Italy on his honeymoon trip when gradually started complaining of upper respiratory symptoms that progressed to shortness of breath and fever with chills. He requested medical opinion in Milan. He was prescribed oral antibiotics with no response. Few days after that he noticed swelling, pain and redness of the right eye along with fluctuating headache. After that he returned to home country for further medical management.

Upon arrival to Salmaniya Medical Complex, he complained of the above mentioned symptoms with the addition of significant photophobia and vision loss of the right eye. He also mentioned noticeable dark urine with urethral discharge. No history of joint pain or genital ulcers.

In the emergency department he was conscious, oriented and not in distress. He had a documented fever of 39.2 degree centigrade but maintained a normal blood pressure and oxygen saturation on room air. On systemic examination, he had reduced air entry bilaterally on lung examination with a diastolic murmur over the mitral valve area. Abdominal and central nervous system examination were not significant for any abnormality. The right eye had severe conjunctival chemosis, clear cornea, exudative membrane over the lens and posterior synichea.

The laboratory investigations showed leuckocytosis with left shift, high ESR, and normal renal and liver function test. The chest X-ray showed bilateral basel infiltrate (see Figure 1).

Figure 1. the chest $x$-ray on admission to the hospital showing bilateral infiltrate along with multiple nodules on the right lung more the left lung

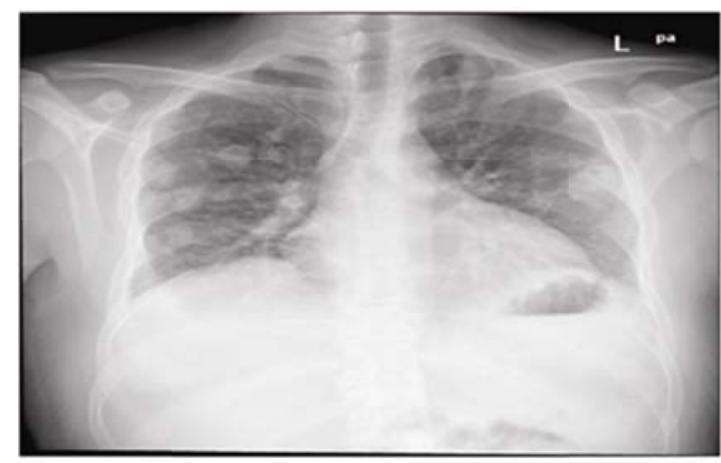

He was admitted with the impression of bilateral pneumonia and right eye uveitis. He was started on Meropenem and Moxifloxacin intravenously plus oral Oseltamivir and several topical eye antibiotics drop. After 48 hours of antibiotics the patient did not show any clinical improvement with continuous spike of fever up to 39 degrees. Upon that a full computed tomography study (CT) was requested which showed the following:

Multiple variable size nodules in both lungs in the upper and lower lobes. Some of these nodules show cavitation with gas within the nodule. The largest measuring $3 \mathrm{~cm}$ with minimal pleural effusion. In the right hepatic lobe segment eight there was well defined irregular wall hypodense lesion. The density value of 10-20 HU within the lesion. The lesion was most consistent with a liver abscess. The rest of the study was non-significant (see Figure 2). 

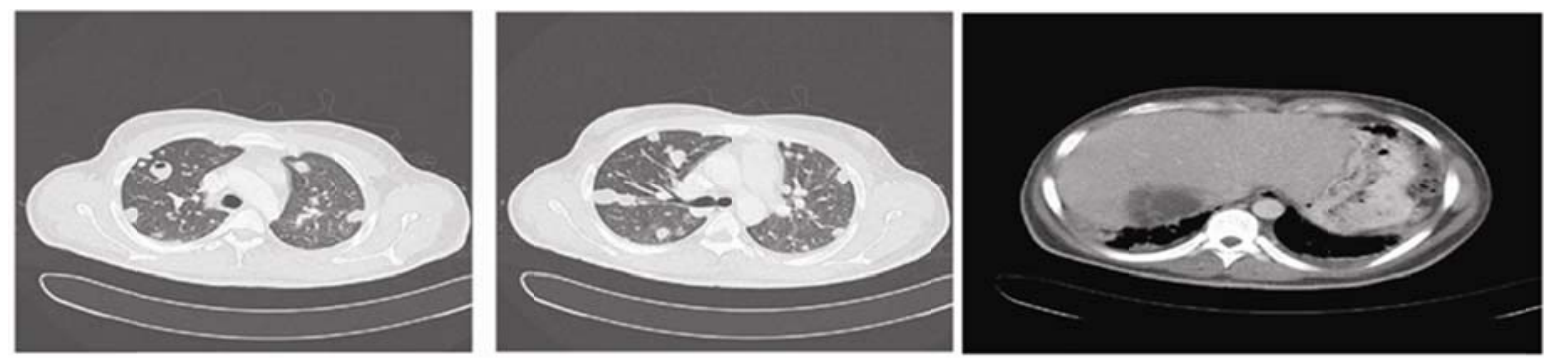

Figure 2. The CT film of abdomen showing the hypodense lesion in the right lobe of the liver. The Chest Cuts of the CT shows the dispersed nodules in both lung fields and the cavitary leasion in the right lung

Based on the radiological study aspiration of the abscess under ultrasound guidance was done. The aspirated fluid was sent for culture and gram stain. Trans-esophageal echocardiogram was done too. It showed normal left ventricular systolic function with ejection fraction $60 \%$. He had mild mitral and tricuspid regurgitation no evidence of vegetation or clots.

B scan ultrasound of the right eye showed severe uveitis, superior posterior vitreous detachment with sub-retinal abscess extending temporally. The finding was suggestive of endophthalmitis.

The following cultures came back as sterile: blood, eye swab, deep tracheal aspiration, and urine. The MERS coronavirus and influenza polymerase chain reaction (PCR) were negative. Respiratory profile for atypical organism and viruses were negative. Mantoux test and vaculitis screen were negative. Mycobacterium tuberculosis PCR in the tracheal aspirate was negative. The culture of the liver aspirate has grown pan sensitive Klebsiella pneumoniae.

After that the Oseltamivir and Vancomycin were stopped and he was continued on Meropenem and Moxifloxacin. With these antibiotics the temperature was gradually subsiding but the severity of the headache was worsening. For that magnetic resonance imaging (MRI) for the brain and orbits was done. It showed extensive infective etiology involving right eyeball with possibly choroiditis? Choroidal detachment associated with periorbital inflammatory soft tissue thickening and small pockets of fluid/abscess as described above. Mild peripheral enhancement of right optic nerve at attachment with eyeball with normal rest of optic nerve. Multiple ring enhancing small lesions involving both cerebral hemispheres and posterior fossa - most likely due to septic emboli (see Figure 3).
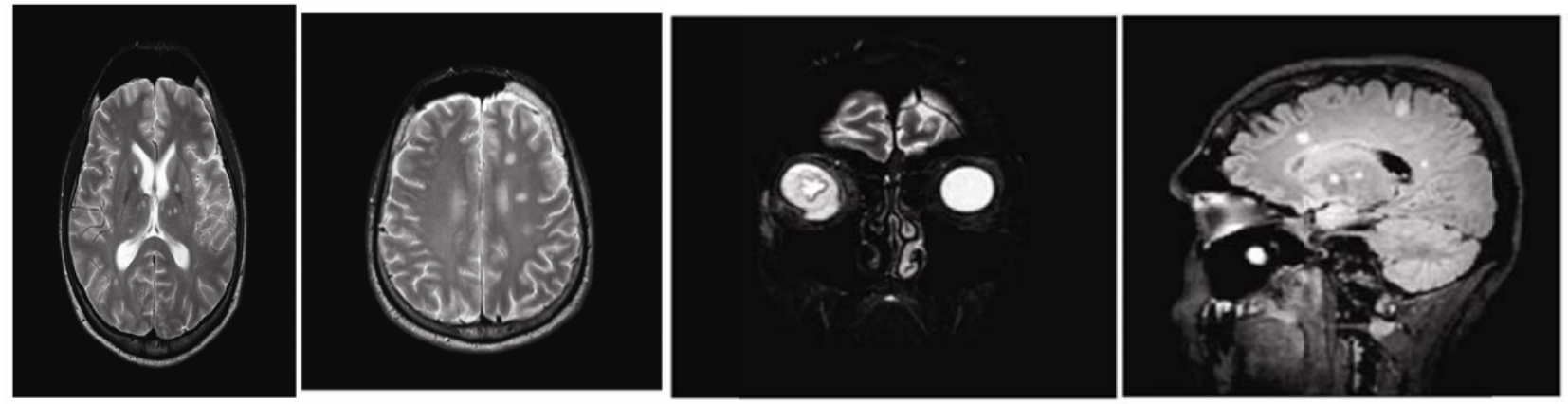

Figure 3. MRI Brain (different views) and orbit showing ring enhancing lesions suggestive of multiple septic emboli with right orbit collection

He received multiple intra-vitreal injections of Vancomycin and Ceftazidime. He was advised for vitrectomy by the ophthalmologist as his vision was not improving but the procedure was deemed by the anesthetist due to the high risk of the procedure. 
The final impression was of Klebsiella pneumonia liver abscess with septic emboli to the brain and right eye. He was continued on multiple antibiotics as mentioned previously but the patient left the hospital seeking second opinion. He had the same diagnosis in the other center and was continued on the same antibiotics which he is still receiving and planning for a prolonged course of antibiotics.

\section{Discussion}

Pyogenic liver abscess is a life threating condition which can be caused by bacteria or fungal infections. Escherichia coli was the leading cause of liver abscesses but recently Klebsiella pneumoniae is emerging as new cause particularly in Asia and Western countries ${ }^{[4]}$. A community-acquired Klebsiella pneumoniae liver abscess syndrome has been recognized in Asia for more than 20 years, with more than 1000 reported cases by $2008^{[5]}$. The reasons for the geographic differences in the incidence of KPLA syndrome are unknown, but hypotheses should address differences in socioeconomic factors, possible occupational exposures (e.g., food handling), 49 defects in host defenses caused by diabetes mellitus or alcoholism, and possibly differences in genetic and immunologic susceptibilities in different ethnic groups ${ }^{[6]}$.

Reports of such infection from North America and Europe are rare. In Alameda County Medical Center in Oakland, California, States , one case occurred in 2006, 3 in 2007, 7 in 2008, and 2 in January 2009. The median age was 52 years (range 37-70 years), and 9 were men. Ten of those patients were born in Asian countries but migrated years earlier to the States and two were born there. Five of the diagnosed patients had no underlying illnesses and seven were known cases of diabetes mellitus, one with alpha thalasemia, two with active malignancy and one with pre-existing multiple organ failure. Those patients had diverse presentation of their infection. Seven had liver abscess, one kidney abscess complicated by multiple brain emboli and pulmonary septic emboli, and two presented with neck abscesses. Nine of the patients the course of illness was complicated by venous thrombosis and four with pulmonary emboli diagnosed via CT scan. Some of the complications were diagnosed upon presenting to the hospital and in other during their treatment of the primary presentation ${ }^{[7]}$.

Other cases reports were documented as well. An example, a 29-year-old Indian male who presented to medical attention with history fever and right upper quadrant pain. The ultrasound and CT scan showed a hypodense lesion which was aspirated but did not grow any organism. However, two blood cultures have grown Klebsiella pneumonia. He was treated with intravenous Metronidazole and Gentamicin. The patient showed good response. Another case of a 78-year-old Chinese male who had fever, chills and right upper quadrant pain. The CT confirmed the presence of a hypodense lesion in the right lobe of the liver. The aspirate of the lesion grew Klebsiella pneumonia and three sets of blood culture grew the same organism. The patient responded very well to post aspiration and discharged on oral levofloxacin and metronidazole for 4 weeks ${ }^{[8]}$.

A retrospective study of 110 cases of primary liver abscess caused by Klebsiella pneumonia that required hospitalization during 2001-2002 was conducted to identify predictors of metastatic infection in Taiwan. The study showed complications including septic shock, acute respiratory failure requiring mechanical ventilation, and metastatic infections. Most of the severe complications occurred within the first $72 \mathrm{~h}$ from presentation to the medical care. Seventeen (15.5\%) of the 110 patients had metastatic infections. Approximately one-third of these received a diagnosis of metastatic infection at the time of presentation. Three-quarters were noted within $72 \mathrm{~h}$ after presentation. These 17 infections included meningitis (11 patients [64.7\%]), endophthalmitis (4 [23.5\%]), septic pulmonary emboli (5 [29.4\%]), and other types (3 [17.6\%]). Among the 4 patients with late metastatic infection, 2 had meningitis that became apparent on days 4 and 15 of hospitalization; 1 had endophthalmitis noted on day 6, and 1 had right-sided empyema noted on day $20^{[9]}$.

So as can be seen from the case reports mentioned above that Klebsiella pneumoniae has a wide variety of presentation where abscesses can be formed in multiple organs and accordingly the mode of presentation can differ. However, those who had a liver abscess had a similar presentation to the patient in our case. It is obvious too that complication can be 
diagnosed at the time of first presentation to the hospital and it can develop even while the patient is on antibiotics. In our case the patient had evidence of septic emboli upon presentation which were aggravating and worsening while he was on antibiotics covering the organism. This leads us to the point that close attention should be paid to the possibility of metastasis all the time and that antibiotics coverage is not enough to prevent it. The susceptibility to the organism and spread of the organism was proven to be higher in those with underlying co-morbidities especially diabetes but it is not a rule of thumb as in our case the patient was leading a completely healthy life until he was diagnosed with the disease.

In conclusion, Klebsiella pneumonia liver abscess is a community acquired organism associated with diabetes and one or more septic emboli. It is not known yet to be resistant to antibiotics, and therefore it usually has a good outcome. Therefore, physicians should be aware of this insidious syndrome and should identify early signs of septic emboli (e.g. meningitis or endophthalmitis) because on time management remains critical for a good outcome.

\section{References}

[1] Ryan KJ, Ray CG, Ahmad N, et al. Sherris Medical Microbiology. 4th ed. New York: McGraw Hill; 2004.

[2] Podschun R, Ullmann U. Klebsiella spp. as Nosocomial Pathogens: Epidemiology, Taxonomy, Typing Methods, and Pathogenicity Factors. Clinical Microbiology Reviews. 1998; 11 (4): 589-603. PMid:9767057.

[3] Siu LK, Yeh KM, Lin JC, et al. Klebsiella pneumoniae liver abscess: a new invasive syndrome. Lancet Infect Dis. 2012; 12(11): 881-7. http://dx.doi.org/10.1016/S1473-3099(12)70205-0

[4] Rahimian J, Wilson T, Oram V, et al. Pyogenic liver abscess: recent trends in etiology and mortality. Clin Infect Dis. 2004; 39: 1654-59. PMid:15578367 http://dx.doi.org/10.1086/425616

[5] McIver CJ, Janda JM. Pathogenesis and laboratory identification of emerging hepatovirulent Klebsiella pneumoniae. Clin Microbiol News. 2008; 30: 127-31. http://dx.doi.org/10.1016/j.clinmicnews.2008.08.001

[6] Habib AG, Tambyah PA. Community-acquired Klebsiella pneumoniae central nervous system infections in adults in Singapore. Eur J Clin Microbiol Infect Dis. 2003; 22(8): 486-8. PMid:12884061 http://dx.doi.org/10.1007/s10096-003-0963-x

[7] Robert MC, Larry L, Brad F. Invasive Klebsiella Pneumoniae infection, California, USA. Emerging Infectious Diseases. 2010; 16(9): 150-8. PMid:20735943 http://dx.doi.org/10.3201/eid1609.100386

[8] Francesco C, Luigi F, Valentina R, et al. Liver abscess caused by Klebsiellapneumoniae: two case reports. Cases Journal. 2009; 2: 6879. PMid:19829875 http://dx.doi.org/10.1186/1757-1626-0002-0000006879

[9] Susan SJ Lee, Chen YS, Tsai HC, et al. Predictors of Septic Metastatic Infection and Mortality among Patients with Klebsiella pneumonia Liver Abscess. Oxford Journals, Medicine \& Health, Clinical Infectious Diseases. 2008; 47(5): 642-50.

PMid:18643760 http://dx.doi.org/10.1086/590932 